\title{
Biomasa de raíces finas en arreglos agroforestales con cacao en la Amazonia occidental colombiana
}

\author{
Biomass of fine roots in agroforestry arrangements with \\ cocoa in the western Colombian Amazon
}

\section{Adrián Rico A. ${ }^{1}$ j Juan Carlos Suárez S. ${ }^{2}$}

1 Profesor, Ingeniero Agroecólogo, M.Sc. Agroforestería, Facultad de Ciencias Agropecuarias, Programa Maestría en Agroforestería, Universidad de la Amazonia, Florencia, Colombia, a.rico@udla.edu.co.

2 Profesor, Ingeniero Agroecólogo, M.Sc. Agroforestería Tropical, Facultad de Ingeniería, Programa Ingeniería Agroecológica, Universidad de la Amazonia, Florencia, Colombia, ju.suarez@udla.edu.co.

Citar: Rico, A. \& Suárez, J. (2018). Biomasa de raíces finas en arreglos agroforestales con cacao en la amazonia occidental colombiana. Rev. Cienc. Agr. 35(1): 26-35. doi: http://dx.doi.org/10.22267/rcia.183501.80.

Recibido: Enero 29 de $2017 . \quad$ Aceptado: Agosto 30 de 2017.

\section{RESUMEN}

Los arreglos agroforestales con cacao poseen una estructura diversificada, la cual influye sobre el comportamiento de las raíces finas, cuya distribución está influenciada por las diferentes prácticas culturales. El objetivo del presente trabajo fue estudiar la distribución, el aporte de biomasa de raíces finas en relación con la densidad aparente y el porcentaje de humedad en arreglos agroforestales con cacao en el Centro de Investigaciones CIMAZ Macagual (1037'LN y 75036'LO). El muestreo se desarrolló en tres arreglos agroforestales con cacao clasificados en sombra intensa, semisombra y pleno sol. Para la extracción de la biomasa de raíces se utilizó el cilindro de volumen conocido cada $10 \mathrm{~cm}$ hasta llegar a $1 \mathrm{~m}$ de profundidad. Las raíces finas fueron extraídas a través de una malla de un tamaño $<2 \mathrm{~mm}$. Se presentaron diferencias estadísticas significativas entre la interacción arreglo-profundidad para la variable biomasa de raíces $(<0,001)$ con una correlación positiva $(0,26)$ entre el peso y la densidad aparente. La mayor acumulación de biomasa de raíces se presentó entre los primeros $20 \mathrm{~cm}$ de profundidad en los tres sistemas estudiados, donde la densidad aparente aumenta en los diferentes perfiles del suelo; a su vez la humedad gravimétrica fue mayor en los arreglos agroforestales que en cacao a pleno sol, mostrando diferencias significativas $(\mathrm{P}<0,001)$ con una correlación negativa $(-0,75)$ en cuanto a las profundidades. En este sentido, los sistemas agroforestales favorecen la disponibilidad de agua en los primeros perfiles del suelo aumentando la presencia de raíces finas.

Palabras clave: Agroforestería, theobroma, sistema radical, densidad aparente, suelo. 


\begin{abstract}
The agroforestry arrangements with cocoa have a diversified structure, which influences the behavior of fine roots, whose distribution is influenced by different cultural practices. The objective of the present work was to study the distribution, the contribution of biomass of fine roots in relation to the apparent density and the percentage of humidity in agroforestry arrangements with cocoa in the CIMAZ Macagual Research Center $\left(1^{\circ} 37^{\prime} \mathrm{LN}\right.$ and $\left.75^{\circ} 36^{\prime} \mathrm{LW}\right)$. The sampling was developed in three agroforestry arrangements with cocoa classified in intense shade, semi-shade and full sun. For the extraction of the biomass of roots the cylinder of known volume was used every $10 \mathrm{~cm}$ until reaching $1 \mathrm{~m}$ of depth. The fine roots were extracted through a mesh size $<2 \mathrm{~mm}$. There were significant statistical differences between the array-depth interaction for the variable root biomass $(<0.001)$ with a positive correlation $(0.26)$ between weight and bulk density. The highest accumulation of root biomass occurred between the first $20 \mathrm{~cm}$ of depth in the three systems studied, where the apparent density increases in the different soil profiles; in turn, the gravimetric humidity was greater in the agroforestry arrangements than in cocoa in full sun, showing significant differences $(\mathrm{P}<0.001)$ with a negative correlation $(-0.75)$ in terms of the depths. In this sense, agroforestry systems favor the availability of water in the first soil profiles, increasing the presence of fine roots.
\end{abstract}

Keywords: Agroforestry, theobroma, root system, apparent density, soil.

\section{INTRODUCCIÓN}

Los cambios a nivel del suelo relacionados con la química y física bajo arreglos agroforestales se debe al desarrollo, descomposición de raíces y materia orgánica (Montagnini y Nair, 2004; Barreto y Leon, 2005; Ehrenbergerová et al., 2015). Es por ello, que es necesario conocer su biomasa, velocidad de crecimiento y distribución de raíces finas. Este tipo de información es importante para el desarrollarlo de diferentes prácticas culturales (aplicaciones de fertilizantes, esquemas de riego) que permitan incrementar la producción, y eviten la competencia interespecífica entre árboles y cultivos por recursos (agua y nutrientes) (George et al., 2009; Sherin et al., 2009).

Para analizar la biomasa, crecimiento y distribución de las raíces, se han clasificado de acuerdo con su diámetro como raíces finas $(<2 \mathrm{~mm})$ y gruesas $(>2 \mathrm{~mm})$ debido a sus diferencias en el desarrollo, mortalidad y función en la toma de agua y nutrientes (Gill y Jackson, 2000). Las raíces gruesas funcionan como anclaje, ejerciendo soporte estructural para la planta (Coutts et al., 1999), pro- porcionando la red de conducción de sustancias asimiladas por las raíces finas. En este caso, las raíces finas son las responsables de la absorción, asimilación de agua y nutrientes en los micro y macro poros del suelo, y conforman la mayor parte de la longitud y superficie radical (Claus y George, 2005; Padilla et al., 2015).

En este sentido, los arreglos agroforestales asociados con cacao, con café, o con cacao y café, poseen una estructura multi-estratificada, donde se puede observar especies de bosque secundario y tener estructuras verticales similares (Guiracocha et al., 2001) que influyen sobre el comportamiento de las raíces finas por la presencia de las diferentes cohortes (alta, media y baja). Este componente puede verse afectado por perturbaciones naturales o antrópicas, logrando recuperarse fácilmente, dependiendo de la magnitud, la extensión, la duración de la perturbación y las condiciones ambientales (Bucheli et al, 2013).

Se ha reportado que la presencia de raíces finas se encuentran en los primeros $20 \mathrm{~cm}$ de profundidad tal como se ha encontrado en asociaciones de café 
- eucalipto (Eucalyptus deglupta) (Schaller et al., 2003) Morales y Beer (1998), café-leguminosas (A. idiopoda y E. poeppigiana) y cacao-leguminosas (Inga edulis) (Gómez et al., 2009).

Por lo tanto, la biomasa de las raíces finas y su distribución, está muy influenciada por el genotipo, el sitio, la textura del suelo, la edad de la planta (Dwyer et al., 1996; Huang et al., 2008), la densidad aparente, la resistencia a la penetración influenciado por las distintas prácticas agronómicas como la fertilización, el riego (Fabião et al., 1995; Bassoi et al., 2003) siendo el agua un factor limitante clave para el crecimiento vegetal (Sayer et al., 2006) y en gran medida en la distribución de raíces finas (Zhou y Shangguan, 2007; Schenk, 2008), la poda (Jones et al., 1998; Steudle, 2000) y las técnicas de labores al suelo (Madeira et al., 1989). El objetivo del estudio fue cuantificar el aporte de biomasa de raíces finas y su relación con la densidad aparente y la humedad gravimétrica a diferentes profundidades en dos estructuras agroforestales con cacao y en cultivo a plena exposición.

\section{MATERIALES Y MÉTODOS}

Sitio de estudio y arreglos agroforestales. El estudio se realizó en el Centro de Investigaciones Macagual $\left(1^{\circ} 37^{\prime} \mathrm{LN}\right.$ y $\left.75^{\circ} 36^{\prime} \mathrm{LO}\right)$ de la Universidad de la Amazonia. Presenta una precipitación promedio de 3793mm, un brillo solar de 1707 horas año $0^{-1}$, temperatura promedio anual de $25,5^{\circ} \mathrm{C}$ y humedad relativa promedio anual de $84,25 \%$. Los arreglos conformados con las plantas de cacao, fueron plantados dirección oriente a occidente, con distancia de siembra de plantas de cacao de $3 \times 3 \mathrm{~m}$.

En cada arreglo agroforestal, se monitoreó el aporte de biomasa de raíces, densidad aparente y humedad gravimétrica tres niveles de sombra: i. Alta densidad de musáceas (Musa paradisiaca, Musa $\mathrm{AAB}$ ) intercalado con cacao (SC), con una densidad de siembra de 127 plantas ha- ${ }^{-1}$, ii. Baja densidad de árboles agregados clasificado como sombra media (SS) y generada por el agrupamiento de árboles, cuyo factor de radiación transmitida promedio fue de $29 \pm 1,8 \%$ y iii. Multiestrato diversificado con un promedio de radiación transmitido de $62,2 \pm 2,2 \%$ clasificado como sombra intensa (SI) (Tabla1).

Tabla 1. Arreglos agroforestales con cacao en el Centro de Investigaciones Cesar Augusto Estrada CIMAZ Macagual.

\begin{tabular}{ll}
\hline $\begin{array}{c}\text { Código } \\
\text { del Sitio }\end{array}$ & \multicolumn{1}{c}{ Descripción } \\
\hline \multirow{3}{*}{ SI } & $\begin{array}{l}\text { Arreglo agroforestal con una som- } \\
\text { bra del } 80 \% \text { compuesto por espe- } \\
\text { cies maderables en la cohorte supe- } \\
\\
\end{array}$ \\
& rior: Capiron (Capirona decorticans \\
& Spruce), Abarco (Cariniana pyrifor- \\
& mis Miers), Marfil (Berchemia disco- \\
& lor (Klotzsch) Hemssl.), Acacia sp. \\
& Intercalado con cacao.
\end{tabular}

Arreglo agroforestal con una sombra del $60 \%$ compuesto por especies maderables en la cohorte superior: Capiron (Capirona decorticans Spruce), Abarco (Cariniana pyriformis Miers), Marfil (Berchemia discolor (Klotzsch), Uva caimarona (Pourouma cecropiifolia Mart.), Maraco (Theobroma bicolor Bonpl.) intercalado con cacao.

Cultivo de cacao sin cubierta de árboSC les (pleno sol).

Densidad aparente y biomasa de raíces. En cada sistema con cacao, se realizaron parcelas de $14 \times 32 \mathrm{~m}$ con tres calicatas $(1 \times 1 \times 1,2 \mathrm{~m})$ distribuidas aleatoriamente. En total, se tomaron 270 muestras de densidad aparente y raíces finas, en dos arreglos agroforestales con cacao y a pleno sol con exposición en tres caras (cara A - cara B cara C) a diez profundidades. 
Para la toma de las muestras, se siguió la metodología del cilindro del volumen conocido $\left(98,17 \mathrm{~cm}^{3}\right)$ con el mismo principio del método del barreno (Bohm, 1979). Una vez tomada la muestra, se llevó al horno a una temperatura de $105^{\circ} \mathrm{C}$ hasta alcanzar peso constante (Upson y Burgess, 2013). Posteriormente, se retiraron las raíces finas presentes y fueron pesadas en una balanza de precisión de $0,001 \mathrm{~g}$ con el fin de ver el aporte de biomasa de raíces finas por profundidad en los diferentes niveles de sombra, las cuales fueron extrapoladas a biomasa por hectárea.

Análisis de datos. Se realizaron Modelos Lineales Generales y Mixtos (MLM) para explicar la biomasa de raíces, densidad aparente y humedad gravimétrica en diferentes arreglos agroforestales de cacao (factor fijo con tres estructuras agroforestales) a diferentes profundidades (cada $10 \mathrm{~cm}$ hasta llegar a $1 \mathrm{~m}$ de profundidad). Como efecto aleatorio se incluyó la repetición. En cada uno de los MLM, los supuestos de normalidad y homogeneidad de varianza fueron evaluados usando los residuos estudentizados. Los análisis se realizaron utilizando el programa InfoStat (Di Rienzo et al., 2015) mediante una interface amigable de la plataforma $\mathrm{R}$ versión 3.3.2 (R Development Core Team, 2017) basado en el paquete $n$ lme (Pinheiro et al., 2015).

\section{RESULTADOS Y DISCUSIÓN}

El mayor aporte de biomasa de raíces fue en los primeros $10 \mathrm{~cm}$ en los tres sistemas (SI, SS, SC) decreciendo al aumentar la profundidad. Se encontró que a la profundidad de $20 \mathrm{~cm}$, el nivel de semisombra (SS) presentó la mayor biomasa radical, a diferencia de las profundidades 40 y $60 \mathrm{~cm}$ en el arreglo sombra intensa (SI) (Figura 1).

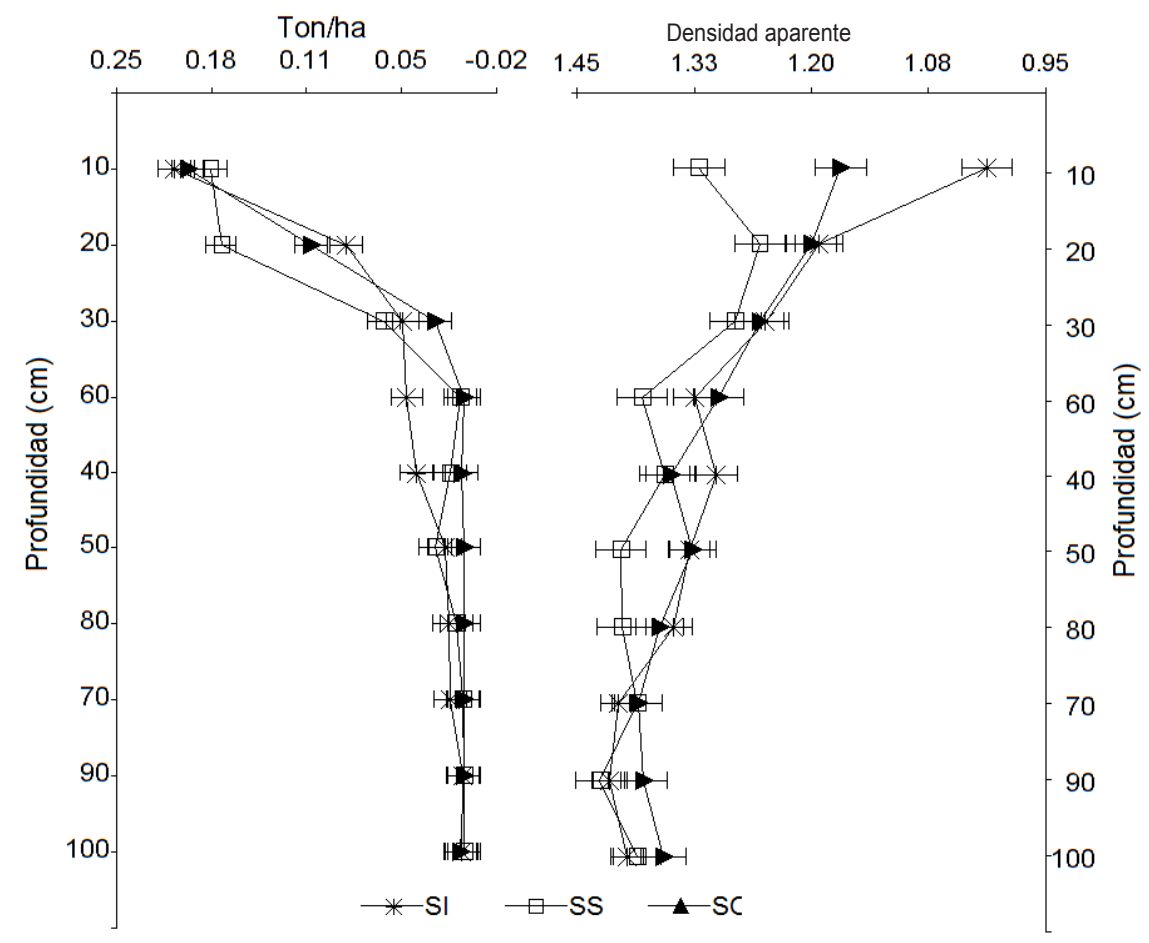

Figura 1. Distribución de la biomasa de raíces finas y densidad aparente en tres niveles sombra en arreglos agroforestales de Theobroma cacao L. 
La interacción arreglo-profundidad fue significativa para la variable biomasa de raíces $(\mathrm{P}<0,001)$ mostrando una correlación positiva $(0,26)$ entre el peso de la biomasa (g) y la densidad aparente. Lo anterior es similar a lo reportado por otros estudios (Bakker et al., 2006; Das y Chaturvedi, 2008; Ma et al., 2013), donde mencionan que la biomasa de las raíces se encuentra principalmente en los primeros veinte centímetros del suelo y tiende a disminuir al aumentar la profundidad (Yanai et al., 2006; Pransiska et al., 2016).

Stokes et al. (2008) indican que la variabilidad observada se debe a la heterogeneidad espacial de desarrollo del sistema radicular, que depende de las interacciones de los factores genéticos y ambientales de los árboles, y puede explicarse por la presencia de diferentes especies de árboles que promueven diferentes condiciones ambientales debido a la distancia de siembra y características morfológicas que influyen en el patrón de variabilidad espacial (Katayama et al., 2009; de Carvalho et al., 2016).

Kang et al. (2010) demostraron que la biomasa de raíces en diferentes profundidades de suelo, indica la capacidad de crecimiento de las plantas y biomasa acumulada en capas específicas del suelo, donde una biomasa acumulada más alta significa una mayor capacidad para absorber agua y nutrientes del suelo en los estratos más superficiales (Claus y George, 2005).

Particularmente, en sistemas agroforestales, la profundidad es muy importante, debido a que el sistema de raíces de los árboles de sombra puede alcanzar los dos metros de profundidad o más (Tscharntke et al., 2011). El cacao, por ejemplo, tiene una alta concentración de raíces en los primeros $60 \mathrm{~cm}$, llegando a $1-2 \mathrm{~m}$ de profundidad (Müller y Gama, 2012).
Por otro lado, la densidad aparente entre los diferentes niveles de sombra (SI, SS, SC) tuvo diferencias en los primeros $10 \mathrm{~cm}$ de profundidad, incrementando la densidad al aumentar las profundidades donde muestra similitud entre los sistemas evaluados. El arreglo SI, presentó menor densidad aparente el cual favorece el desarrollo radical en las diferentes profundidades (Figura 1) por lo que mejora la estructura del suelo y la porosidad (Ling et al., 2017).

En cuanto a lo encontrado por Tumwebaze y Byakagaba (2016) en sistemas agroforestales de café y en monocultivo, mencionan que en condiciones de monocultivo de café presentó menor densidad aparente $\left(1,162 \mathrm{mg} / \mathrm{m}^{3}\right)$ en comparación con los sistemas agroforestales de café, cuya densidad aparente osciló entre 1,267 a 1,298mg/m³ donde se incrementa significativamente con el aumento de la profundidad del suelo. Por su parte, Ingaramo et al. (2003) y Henríquez et al. (2011) establecieron la relación directa que existe entre la penetración de raíces con la densidad del suelo, donde un aumento en la densidad aparente indica disminución del espacio poroso total y disminución del desarrollo radical.

En relación con la humedad gravimétrica y la densidad aparente, se obtuvieron diferencias significativas y una correlación negativa $(-0,75)$ en cuanto a las profundidades. Se presentó una diferencia entre los arreglos (SS, SI) y (SC) en los primeros $80 \mathrm{~cm}$ de profundidad respecto al porcentaje de humedad. SS fue el de mayor retención en las diferentes profundidades a diferencia del SC, en el cual se refleja una menor retención de humedad en estas profundidades (Figura 2). Los resultados obtenidos contrastan con los de Hossne y Salazar (2004), quienes mostraron una retención de humedad de 7,68 a $12,01 \%$, aumentando al profundizar en el perfil del suelo. 


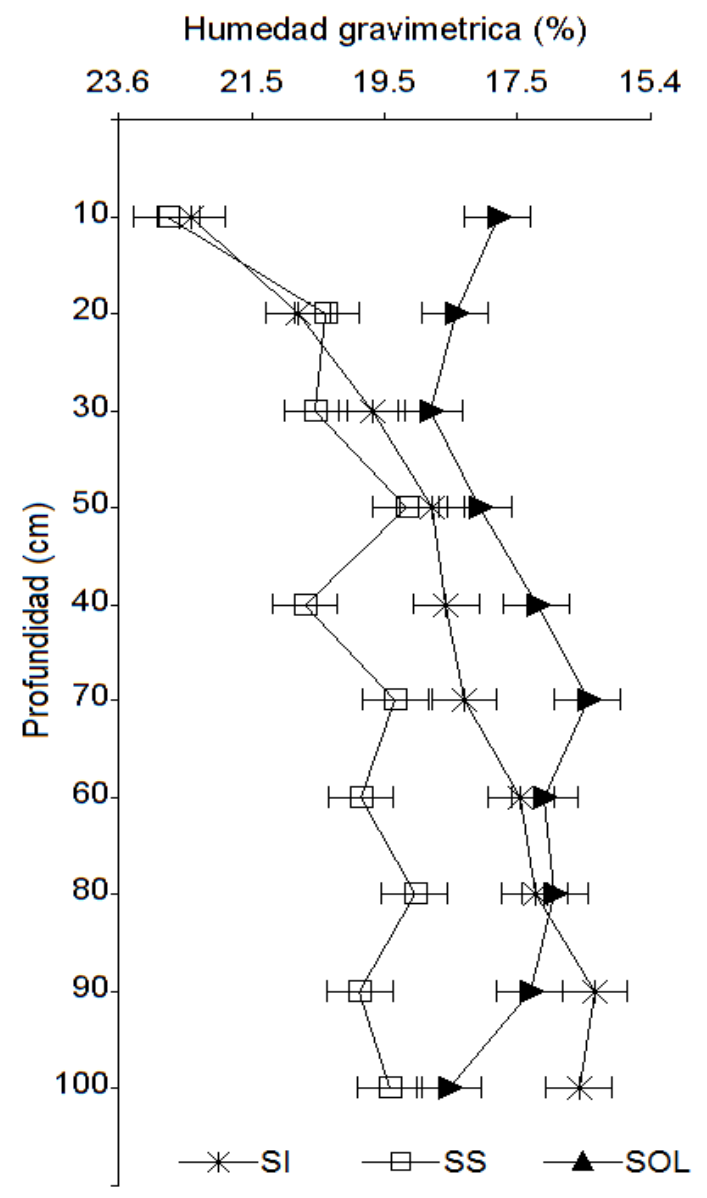

Figura 2. Humedad gravimétrica en tres niveles de sombra en arreglos agroforestales de cacao en el Centro de Investigaciones Cesar Augusto Estrada CIMAZ Macagual.

Sands y Mulligan (1990) mencionan que la densidad de raíces es mayor cerca a la superficie del suelo, decreciendo exponencialmente con la profundidad. Gaitán et al. (2005) encontraron que la distribución de las raíces finas de Eucalyptus globulus ssp. Maidenii está estrechamente relaciona con las propiedades físicas y químicas del suelo, donde el crecimiento radical se ve favorecido en los primeros centímetros del suelo, así mismo, la disponibilidad de nutrientes, la resistencia mecánica, la aireación y la temperatura son más favorables a menor profundidad. Sin embargo, la distribución del sistema radical puede ser modificada por cambios en las condiciones edáficas, especialmente en la distribución de agua, nutrientes y temperatura (Zadworny et al., 2017). Según Cronan (2003), estimaciones de biomasa radical basada en muestreos arrojarían diferentes resultados dependiendo de la metodología utilizada, tanto referido al tamaño de la muestra, a la profundidad de muestreo, como a la época del año en que se extrae la muestra, sumándose a estas variaciones el error de procesamiento de la muestra (Nakahata y Osawa, 2017). 


\section{CONCLUSIONES}

Las propiedades físicas del suelo como la densidad aparente, la compactación y la humedad gravimétrica a lo largo del perfil, influyen en la distribución vertical y la cantidad de las raíces finas en los arreglos estudiados. En este sentido, la densidad aparente $(d a)$ afectó la biomasa de raíces. Los sistemas agroforestales modifican las condiciones microclimáticas, por ende reducen la temperatura favoreciendo la retención y disponibilidad de agua en el sistema.

Las raíces bajo cacaotales se encontraron en los primeros $10 \mathrm{~cm}$ de profundidad presentando un mayor aporte de biomasa bajo los arreglos agroforestales comparado con el monocultivo.

Conflicto de intereses: Los autores declaran que no hay conflicto de interés.

\section{REFERENCIAS BIBLIOGRÁFICAS}

Bakker, E. G., Stahl, E. A., Toomajian, C., Nordborg, M., Kreitman, M. \& Bergelson, J. (2006). Distribution of genetic variation within and among local populations of Arabidopsis thaliana over its species range. Molecular Ecology. 15(5): 1405-1418. doi: 10.1111/j.1365-294X.2006.02884.x.

Barreto, L.H. \& León, J.D. (2005). Masa total y contenido de nutrientes en raíces finas de ecosistemas forestales (Pinus patula Schltdl y Cham., Cupressus lusitánica Mill. Y Quercus humboldtii Bonpl.) de Piedras Blancas, Antioquia-Colombia. Rev. Fac. Nal. Agr. (58): 2907-2929.

Bassoi, L. H., Hopmans, J. W., Jorge, L. A. \& Alencar, C. M. (2003). Grapevine root distribution in drip and micro sprinkler irrigation. Scientia Agricola. 60(2): 377-387. doi: http://dx.doi.org/10.1590/S010390162003000200024.

Bohm, W. (1979). Methods of studying root systems. Berlin: Springer. 140p.
Bucheli, P., Benjamin, T., Rusch, G. M., Ibrahim, M., Casals, P. \& Pugnaire, F. (2013). Estrategias de los árboles en SSP en el uso eficiente del agua y la tolerancia a la sequía, pp. 25 - 30. En: Sánchez, D.; Villanueva, C.; Rusch, G.Ibrahim, M.; DeClerck, F. Estado del Recurso Arbóreo en Fincas Ganaderas y su Contribución en la Sostenibilidad de la Producción en Rivas. Primera edición. Nicaragua. Turrialba, Costa Rica: CATIE. 50p.

Claus, A. \& George, E. (2005). Effect of stand age on fine-root biomass and biomass distribution in three European forest chronosequences. Canadian Journal of Forest Research. 35(7): 1617-1625. doi: https://doi.org/10.1139/x05-079.

Coutts, M. P., Nielsen, C. N. \& Nicoll, B. C. (1999). The development of symmetry, rigidity and anchorage in the structural root system of conifers. Plant and soil. 217(1-2): 1-15.

Cronan, C. S. (2003). Belowground biomass, production, and carbon cycling in mature Norway spruce, Maine, USA. Canadian Journal of Forest Research. 33(2): 339-350. doi: https://doi.org/10.1139/x02-189.

Das, D. K. \& Chaturvedi, O. P. (2008). Root biomass and distribution of five agroforestry tree species. Agroforestry systems. 74(3): 223-230. doi: https:// doi.org/10.1007/s10457-008-9159-9.

De Carvalho, G. L., Cardoso, I. M., De Sá Mendonça, E., Fernandes, R. B. A., Lopes, V. S. \& Oliveira, T. S. (2016). Trees modify the dynamics of soil $\mathrm{CO}_{2}$ efflux in coffee agroforestry systems. Agricultural and Forest Meteorology. 224: 30-39. doi: https://doi. org/10.1016/j.agrformet.2016.05.001.

Di Rienzo, J.A., Casanoves, F., Balzarini, M.G., González, L., Tablada, M. \& Robledo, C.W. (2015). InfoStat versión 2016. Grupo InfoStat, FCA. Argentina: Universidad Nacional de Córdoba.

Dwyer, L. M., MA, B. L., Stewart, D. W., Hayhoe, H. N., Balchin, D., Culley, J. L. B. \& Mcgovern, M. (1996). Root mass distribution under conventional and conservation tillage. Canadian Journal of Soil Science. 76(1): 23-28. doi: https://doi.org/10.4141/cjss96004. 
Ehrenbergerová, L., Cienciala, E., Kučera, A., Guy, L. \& Habrová, H. (2015). Carbon stock in agroforestry coffee plantations with different shade trees in Villa Rica, Peru. Agrofor. Syst. 90(3): 433-445. doi: https://doi.org/10.1007/s10457-015-9865-z.

Fabião, A., Madeira, M., Steen, E., Kätterer, T., Ribeiro, C. \& Araújo, C. (1995). Development of root biomass in an Eucalyptus globulus plantation under different water and nutrient regimes. Plant and Soil. 168(1): 215-223. doi: https://doi.org/10.1007/978-94011-0455-5_24.

Gaitán, J. J., Penón, E. A. \& Costa, M. C. (2005). Distribución de raíces finas de Eucalyptus globulus ssp. maidenii y su relación con algunas propiedades del suelo. Ciência Florestal. 15(1): 33-41.

George, S., Suresh, P. R., Wahid, P. A., Nair, R. B. \& Punnoose, K. I. (2009). Active root distribution pattern of Hevea brasiliensis determined by radioassay of latex serum. Agroforestry systems. 76(2): 275-281. doi: https://doi.org/10.1007/s10457-008-9104-y.

Gill, R. A. \& Jackson, R. B. (2000). Global patterns of root turnover for terrestrial ecosystems. New Phytologist. 147(1): 13-31.

Gómez, C.A., Leblanc, H. \& Nygren, P. (2009). Distribución de raíces finas de Inga edulis y Theobroma cacao en el suelo de un sistema agroforestal orgánico. Tierra Tropical. 5(2): 141-151.

Guiracocha, G., Harvey, C., Somarriba, E., Krauss, U. \& Carrillo, E. (2001). Conservación de la biodiversidad en sistemas agroforestales con cacao y banano en Talamanca, Costa Rica. Agroforestería en las Américas. 8(30): 7-11.

Henríquez, C., Ortiz, O., Largaespada, K., Portuguéz, P., Vargas, M., Villalobos, P. \& Gómez, D. (2011). Determinación de la resistencia a la penetración, al corte tangencial, densidad aparente y temperatura en un suelo cafetalero, Juan Viñas, Costa Rica. Agronomía costarricense. 35(1): 175-184.

Hossne, A. \& Salazar, J. (2004). Límites de consistencia y sus implicaciones agrícolas en un suelo ultisol de sabana del Estado Monagas de Venezuela. Agronomía Costarricense. 28(1): 69-80.
Huang, G., Zhao, X. Y., Su, Y. G., Zhao, H. L. \& Zhang, T. H. (2008). Vertical distribution, biomass, production and turnover of fine roots along a topographical gradient in a sandy shrubland. Plant and soil. 308(12): 201-212.

Ingaramo, 0., Paz, A. \& Dugo, M. (2003). Evaluación de la densidad aparente en diferentes sistemas de laboreos de suelo, en el NO de la Península Ibérica. Argentina: Comunicaciones Científicas y tecnológicas. Universidad Nacional del Nordeste. 4p.

Jones, M., Sinclair, F. L. \& Grime, V. L. (1998). Effect of tree species and crown pruning on root length and soil water content in semi-arid agroforestry. Plant and soil. 201(2): 197-207.

Kang, B. W., Liu, J. J., Sun, J. H. \& Li, Y. F. (2010). Study on Root Distribution of Artemisa Ordosica in Mu Us Sandy Land. Research of Soil and Water Conservation. 17(4): 119-123. doi: https://doi.org/10.5468/ kjog.2010.53.2.119.

Katayama, A., Kume, T., Komatsu, H., Ohashi, M., Nakagawa, M., Yamashita, M., Otsuki, K., Suzuki, M. \& Kumagai, T. 0. (2009). Effect of forest structure on the spatial variation in soil respiration in a Bornean tropical rainforest. Agricultural and Forest Meteorology. 149(10): 1666-1673. doi: https://doi. org/10.1016/j.agrformet.2009.05.007.

Ling, Q., Gao, X., Zhao, X., Huang, J., Li, H., Li, L., Sun, W. $\&$ Wu, P. (2017). Soil water effects of agroforestry in rainfed jujube (Ziziphus jujube Mill.) orchards on loess hillslopes in Northwest China. Agriculture. Ecosystems \& Environment. 247: 343-351. doi: https://doi.org/10.1016/j.agee.2017.06.031.

Ma, L. H., Liu, X. L. \& Wang, Y. K. (2013). Effects of drip irrigation on deep root distribution, rooting depth, and soil water profile of jujube in a semiarid region. Plant and soil. 373(1-2): 995-1006. doi: https://doi.org/10.1007/s11104-013-1880-0.

Madeira, M. V. A., Melo, M. G., Alexandre, C. A. \& Steen, E. (1989). Effects of deep ploughing and superficial disc harrowing on physical and chemical soil properties and biomass in a new plantation of $E u$ calyptus globulus. Soil and Tillage Research. 14(2): 163-175. 
Montagnini, F. \& Nair, P.K.R. (2004). Carbon sequestration: an underexploited environmental benefit of agroforestry systems. Agrofor. Syst. 61(1-3): 281295.

Morales, E. \& Beer, J. (1998). Distribución de raíces finas de Coffea arabica y Eucalyptus deglupta en cafetales del Valle Central de Costa Rica. Agroforesteria de Las Américas. 5(17/18): 44-48.

Müller, M.W. \& Gama-Rodrígues, A.C. (2012). Cacao agroforestry systems. In: Valle, R.R., Science, Technology and Management of Cacao Tree Brasília. CEPLAC/CEPEC. 246 - 271.

Nakahata, R. \& Osawa, A. (2017). Fine root dynamics after soil disturbance evaluated with a root scanner method. Plant and Soil. 1-21. doi: https://doi. org/10.1007/s11104-017-3361-3.

Padilla, F. M., De Dios Miranda, J., Armas, C. \& Pugnaire, F. I. (2015). Effects of changes in rainfall amount and pattern on root dynamics in an arid shrubland. Journal of Arid Environments. 114: 49-53. doi: https:// doi.org/10.1016/j.jaridenv.2014.11.005.

Pinheiro, D. A., Cavero, B. A. S., Vargas, L., Braccini, G. L., Yoshioka, E. T. 0., Oliveira, M. S. B. \& Tavares-Dias, M. (2015). Performance, parasitic infections, hematology and hepatic histology of Colossoma macropomum (tambaqui) fed on homeopathic product. African Journal of Pharmacy and Pharmacology. 9(4): 82-90. doi: https://doi.org/10.5897/AJPP2014. 4194

Pransiska, Y., Triadiati, T., Tjitrosoedirjo, S., Hertel, D. \& Kotowska, M. M. (2016). Forest conversion impacts on the fine and coarse root system, and soil organic matter in tropical lowlands of Sumatera (Indonesia). Forest Ecology and Management. 379, 288-298. doi: https://doi.org/10.1016/j.foreco.2016.07.038.

R Development Core Team. (2017). R: A Language and Environment for Statistical Computing. Vienna, Austria: R Foundation for Statistical Computing. 2630p.

Sands, R. \& Mulligan, D. R. (1990). Water and nutrient dynamics and tree growth. Forest Ecology and Management. 30(1): 91-111.
Sayer, E. J., Tanner, E. V. J. \& Cheesman, A. W. (2006). Increased litterfall changes fine root distribution in a moist tropical forest. Plant and Soil. 281(1-2): 5-13.

Schaller, M., Schroth, G., Beer, J. \& Jiménez, F. (2003). Species and site characteristics that permit the association of fast-growing trees with crops: the case of Eucalyptus deglupta as coffee shade in Costa Rica. Forest Ecology and Management. 175(1): 205-215.

Schenk, H. J. (2008). Soil depth, plant rooting strategies and species' niches. New Phytologist. 178(2): 223-225.

Sherin, G., Suresh, P., Wahid, P., Nair, R. \& Punnoose, K. (2009). Active root distribution pattern of Hevea brasiliensis determined by radioassay of latex serum. Agroforest Syst. 76:275-281. doi: https:// doi.org/10.1007/s10457-008-9104-y.

Steudle, E. (2000). Water uptake by plant roots: an integration of views. Plant and Soil. 226(1): 45-56.

Stokes, A., Norris, J. E., Van Beek, L. P. H., Bogaard, T., Cammeraat, E., Mickovski, S. B.\& Fourcaud, T. (2008). How vegetation reinforces soil on slopes. In: Slope stability and erosion control: ecotechnological solutions. 65-118, Netherlands: Springer.

Tscharntke, T., Clough, Y., Bhagwat, S.A., Buchori, D., Faust, H., Hertel, D., Hölscher, D., Juhrbandt, J., Kessler, M., Perfecto, I., Scherber, C., Schroth, G., Veldkamp, E. \& Wanger, T.C. (2011). Multifunctional shade-tree management in tropical agroforestry landscapes-areview. Journal of Applied Ecology. 48(3): 619-629. doi: https://doi.org/10.1111/ j.1365-2664.2010.01939.x.

Tumwebaze, S. B. \& Byakagaba, P. (2016). Soil organic carbon stocks under coffee agroforestry systems and coffee monoculture in Uganda. Agriculture. Ecosystems \& Environment. 216: 188-193. doi: https://doi.org/10.1016/j.agee.2015.09.037.

Upson, M. A. \& Burgess, P. J. (2013). Soil organic carbon and root distribution in a temperate arable agroforestry system. Plant and soil. 373(1-2): 43-58. doi: https://doi.org/10.1007/s11104-013-1733-x 
Yanai, R. D., Park, B. B. \& Hamburg, S. P. (2006). The vertical and horizontal distribution of roots in northern hardwood stands of varying age. Canadian journal of forest research. 36(2): 450-459.

Zadworny, M., Mccormack, ML., Zytkowiak, R., Karolewski, P., Mucha, J. \& Oleksyn, J. (2017). Patterns of structural and defense investments in fine roots of Scots pine (Pinus sylvestris L.) across a strong temperature and latitudinal gradient in Europe. Global Change Biol. 23: 1218-1231. doi: https://doi.org/10.1111/gcb.13514.

Zhou, Z. \& Shangguan, Z. (2007). Vertical distribution of fine roots in relation to soil factors in Pinus tabulaeformis Carr. forest of the Loess Plateau of China. Plant and Soil. 291(1-2): 119-129. 